\title{
Damping of power system oscillations using adaptive thyristor-controlled series compensators tuned by artificial neural networks
}

\author{
Y.-Y.Hsu and T.-S.Luor
}

\begin{abstract}
A proportional-integral (PI) controller is designed for thyristor-controlled series compensators (TCSCs) to improve the damping for power system oscillations. To maintain a good damping characteristic over a wide range of operating conditions, the gains of the PI controller are adapted in real time, based on online measured transmission line loadings (real and reactive power flows). To speed up the online gain adaptation process, an artificial neural network which is capable of performing complicated computations in a parallel, distributed manner is designed. A major feature of the proposed adaptive PI controller is that only physically measurable variables (real and reactive power flows over the transmission line) are employed as inputs to the adaptive controller. To demonstrate the effectiveness of the proposed adaptive TCSC controller, computer simulations are performed on a power system under disturbance conditions. It is concluded from the simulation results that the proposed adaptive TCSC controller can yield satisfactory dynamic responses over a wide range of operating conditions. Low-frequency oscillations in the frequency range $0.3-2 \mathrm{~Hz}$ have been effectively damped by the proposed compensators.
\end{abstract}

\section{Introduction}

The application of thyristor-controlled series compensators (TCSCs) to increase the power transfer capability of transmission lines has received much attention in recent years $[1,2]$. The effect of TCSCs on the performance of a power system has been examined from a steady-state as well as a dynamic viewpoint $[1,2]$. In the present work, the way in which power system stability is affected by TCSCs as a result of modulation on the power flow over a transmission line will be of major concern $[3,4]$.

Numerous works on the effect of TCSCs on power system stability have been reported recently [3-15]. The basic concept of how the damping of power system oscillations is affected by TCSC power modulation is given in $[5,6]$. The state feedback controller [7], variable-structure controller [8] and fuzzy logic controller [9-11] were designed for the TCSC to improve the stability of the power system.

Note that generator rotor angle, angular speed and other variables which are normally not available at the TCSC substation were employed as inputs to the TCSC controller in [5-10]. To estimate the required phase/speed states from the measured real power flow at the location of the TCSC, a signal conditioning system comprising reset filters and integrators was developed in [11]. In [12, 13], a pair of remote voltages were synthesised using the voltages and currents measured at the terminals of a TCSC and the Thevenin impedances. Then the synthesised angle signal

(1) IEE, 1999

IEE Proceedings online no. 19990069

DOI: 10.1049/ip-gtd:19990069

Paper first received 5th June and in revised form 30th September 1998

The authors are with the Department of Electrical Engineering, National Taiwan University, Taipei, Taiwan was computed as the difference between the phase angles of the two synthesised voltages. This angle signal was employed for damping control. In a recent work by Choi $e t$ al. [14], a performance index was defined to quantify the overall power imbalance in the system and a state estimator was needed to determine the rate of change of this performance index with respect to the TCSC reactance.

In contrast to the works in [11--14] which required a signal conditioning system, voltage synthesiser or estimator to generate the control inputs to the TCSC, deMello [15] examined the control of TCSC reactance using the rate of change of the measured throughpower in series branches as the controller input. Analysis and simulation results indicated that the damping had been improved by the proposed rate feedback controller.

In the present work, an output feedback proportionalintegral (PI) TCSC controller is designed. As in [15], only the measured real and reactive power flows through the transmission line which are available at the TCSC substation will be employed as inputs to the TCSC controller. Unlike the TCSC controller in [15], the controller gain of which was designed based on a nominal operating condition and remained fixed no matter how the system operating conditions and line loadings changed, the controller gains in the proposed PI controller are adapted in real time based on the system operating conditions. The main reason for adapting the controller gains in real time is to keep the same damping effect under different loading conditions. In the literature, adaptive controllers or self-tuning controllers for both static VAR compensators (SVCs) and power system stabilisers (PSSs) have been applied to improve the damping of low frequency oscillations $[16,17]$. A major disadvantage of these adaptive controllers is that system identification and gain determination require much computational effort in real time. To overcome this difficulty, artificial neural networks (ANNs) which are capable 
of performing complicated computations in a highly distributed, parallel manner have been employed to tune the parameters of PSSs and SVCs in a very efficient manner $[18,19]$.

In the present work, the controller gains of the adaptive PI controller for the TCSC are determined by an ANN. The inputs to the ANN include the measured real and reactive power flows through the transmission line and the outputs from the ANN are the desired PI controller gains. To demonstrate the effectiveness of the proposed adaptive control scheme based on ANN, time-domain simulations are performed on a power system equipped with various controllers, including an adaptive TCSC controller, fixed-gain TCSC controller, adaptive PSS and fixed-gain PSS. It is concluded from computer simulation results that the system damping characteristic can be improved by the proposed adaptive TCSC controller over a wide range of operating conditions. Low-frequency oscillations in the frequency range $0.3-2 \mathrm{~Hz}$ have been effectively damped by the proposed compensators.

\section{System model}

The system under study is a synchronous generator connected to a large power system through a transmission line equipped with a thyristor-controlled series compensator (TCSC) as shown in Fig. 1. The variable series compensation $X_{\text {TCSC }}$ provided by the TCSC is controlled by a TCSC controller.

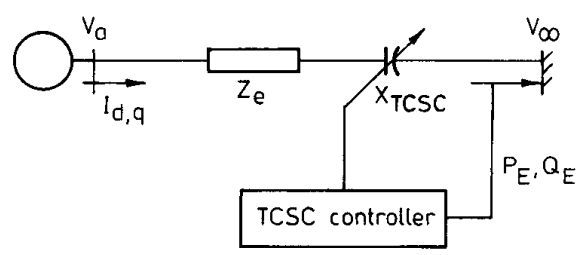

Fig.1 System configuration for synchronous machine connected to large power system through transmission line with TCSC

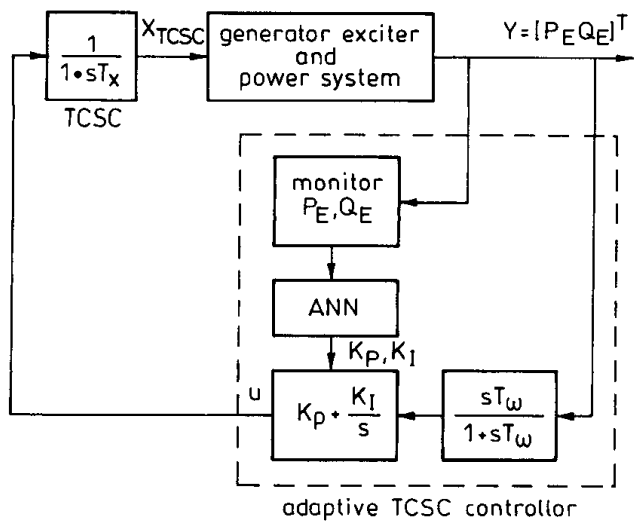

Fig.2 Block diagram of power system with proposed adaptive TCSC controller

Fig. 2 shows a block diagram of the generator, exciter and power system and the proposed artificial neural network based adaptive TCSC controller. The TCSC and its controller can be described by a set of three differential equations.

The TCSC controller is a proportional-integral (PI) controller with the real power $P_{E}$ over the transmission lines as its input. To keep good damping effects under disturbance conditions, the controller gains $K_{P}$ and $K_{l}$ are adapted in real time by the ANN, based on the online-measured real power $P_{E}$ and reactive power $Q_{E}$ over the transmission line.

To simulate the dynamic responses of the system under disturbance conditions, a complete nonlinear generator model [20] is used. The model is described by a set of seven nonlinear differential equations. Details for the seven differential equations can be found in [20]. The excitation system used in this work is an IEEE type 1S system which can be described by two first-order differential equations [20].

The overall model of the generator, exciter and power system equipped with a TCSC controller can be described by the following equations:

$$
\begin{gathered}
\dot{X}(t)=f(X(t), u(t)) \\
Y(t)=g(X(t))
\end{gathered}
$$

where $X(t)$ is the state vector, $u(t)$ is the control signal $X_{T C S C}$ and $Y(t)=\left[P_{E}\right]$ is the output vector.

The power system stabiliser used for comparison with the proposed controller is also a proportional-integral controller with the generator speed deviation as its input.

\section{Design of fixed gain controller}

In the design of a PI TCSC controller, first the nonlinear equations of the study system are linearised around a nominal operating condition, and then the pole assignment method in modal control theory [21] is applied to determine a proper set of controller parameters $T_{w}, K_{p}$ and $K_{I}$ such that the oscillatory mode can be effectively damped.

The linearised nonlinear equations are of the following form:

$$
\begin{gathered}
\dot{X}(t)=A X(t)+B u(t) \\
Y(t)=C X(t)
\end{gathered}
$$

Then, the eigenvalues of the system without a controller can be computed and are summarised in the first column of Table 1.

Table 1: System eigenvalues at $P=1.0, Q=0.6, V_{t}=1.0$

\begin{tabular}{lll}
\hline Open-loop system & With fixed-gain TCSC & With fixed-gain PSS \\
\hline$-0.704 \pm \beta 376.953$ & $-26.399 \pm j 32.311$ & $-0.704 \pm j 376.953$ \\
$-6.845 \pm j 21.699$ & $-48.405 \pm j 4.580$ & $-2.651 \pm j 19.980$ \\
$0.135 \pm j 9.953$ & $-4.5 \pm j 10.681$ & $-4.5 \pm j 10.681$ \\
-47.88 & $-5.6 \pm j 20.037$ & -47.63 \\
-44.73 & -437.29 & -44.18 \\
& -2.4 & -1.92 \\
& -1.01 & -1 \\
\hline
\end{tabular}

It is observed from Table 1 that the electromechanical mode (characterised by the pair of eigenvalues $0.135 \pm$ j9.953) is negatively damped and the eigenvalues for this mode should be shifted leftward to more desirable locations by a PI TCSC controller or a PI PSS. If the pair of eigenvalues $-4.5 \pm j 10.681$ are selected as the desired locations, the TCSC controller gains can be determined as $K_{p}=$ 0.178503 and $K_{I}=-0.00802$. The PSS gains can also be determined as $K_{p}=10.906$ and $K_{I}=0.0764$ under the operating condition of $P=1.0$ and $Q=0.6$.

The eigenvalues for the system equipped with the TCSC controller and the PSS are listed in columns 2 and 3 of Table 1, respectively. It is observed from Table 1 that damping for the electromechanical mode has been improved to a great extent by both TCSC and PSS. 


\section{Application of ANN to adapt TCSC controller gains}

The multilayer feedforward artificial neural network shown in Fig. 3 [22] will be used in this work to adapt the controller gains of both the PI TCSC and PI PSS in real time. Detailed descriptions of this neural network and its application to power systems can be found in $[18,23]$. Before the ANN can be used to adapt the controller gain in real time, it is necessary to determine a proper set of values for the connection weights $w_{i j}$. The process of reaching the connection weights is normally carried out offline and is usually referred to as the training process. In the training process, we first compile a set of training patterns and store these training patterns in the training set. Each training pattern comprises a set of input data and the corresponding output data. In the present work, the measured real power flow $P_{E}$ and reactive power flow $Q_{E}$ through the transmission line are used as the inputs to the ANN. For a pair of $P_{E}$ and $Q_{E}$, we can proceed to determine a set of PI controller gains $\left(K_{p}\right.$ and $\left.K_{I}\right)$ using the pole assignment method, and the results are employed as the ANN output. It is noted that the inputs to the ANN must be replaced by generator real power $P$ and reactive power $Q$ when the ANN is used to adapt PI controller gains for the PSS.

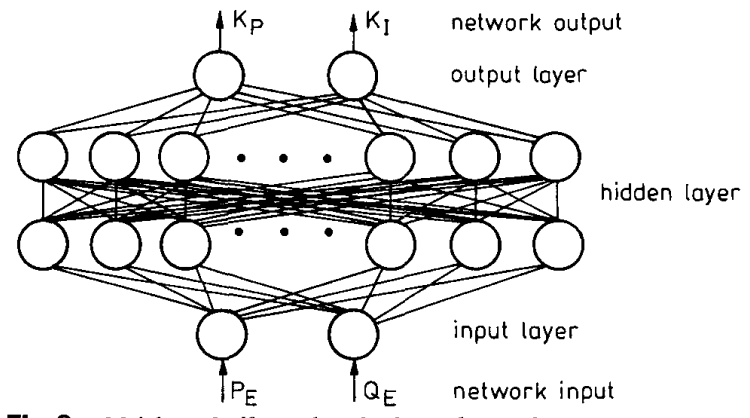

Fig.3 Multilayer feedforward artificial neural network

As mentioned in the previous Section, for the open-loop system, the electromechanical mode is poorly damped. Therefore, in creating training patterns for training the ANN, this mode is shifted to the region on the complex plan as described below:

$$
-4.5 \leq \operatorname{Re}(\lambda) \leq-1.0 \quad \operatorname{Im}(\lambda)=10.681
$$

where the lower bound of the region is selected to be the same as that in the fixed-gain design procedure for comparison. After assigned poles have been determined, the following process can be employed to create training patterns and to train neural networks for both the TCSC and PSS.

For every possible combination of $P_{E}(P)$ and $Q_{E}(Q)$ within the region of interest $\left(0.6 \leq P_{E}(P) \leq 1.5,-0.4 \leq\right.$ $Q_{E}(Q) \leq 1.0$ ), we can compute a pair of desired controller gains. If it is impossible to find a proper pair of controller gains for a certain operating point, it is recommended that the real part and imaginary part of the eigenvalues as described in eqn. 5 be modified. However, the range specified in eqn. 5 works well in all the test cases in this study. The real power and reactive power and the corresponding PI controller gains for any operating condition constitute a training pattern. These training patterns are stored in the training pattern set for the purpose of determining the connection weights. To obtain robust control with a satisfactory damping effect, the training patterns must be chosen to cover all possible network conditions. If the trained ANN fails to give a satisfactory damping effect under certain operating conditions, it is necessary to retrain the
ANN using more training patterns. It should be noted that we use two hidden layers with fifteen hidden nodes per layer in the present work. The momentum constant $\alpha$ is fixed at 0.9 and an adaptive learning rate as described in [23] is employed.

\section{Simulation results}

To demonstrate the effectiveness of the proposed adaptive TCSC controller, the dynamic responses of the system subject to a 0.1 p.u. step change in mechanical torque $\Delta T_{m}$ lasting for five cycles are examined. Fig. 4 illustrates the response curves of the system without PSS, TCSC and TCSC controller under the operating conditions $P=$ 1.0p.u., $Q=0.6$ p.u. and $V_{t}=1.0$ p.u.

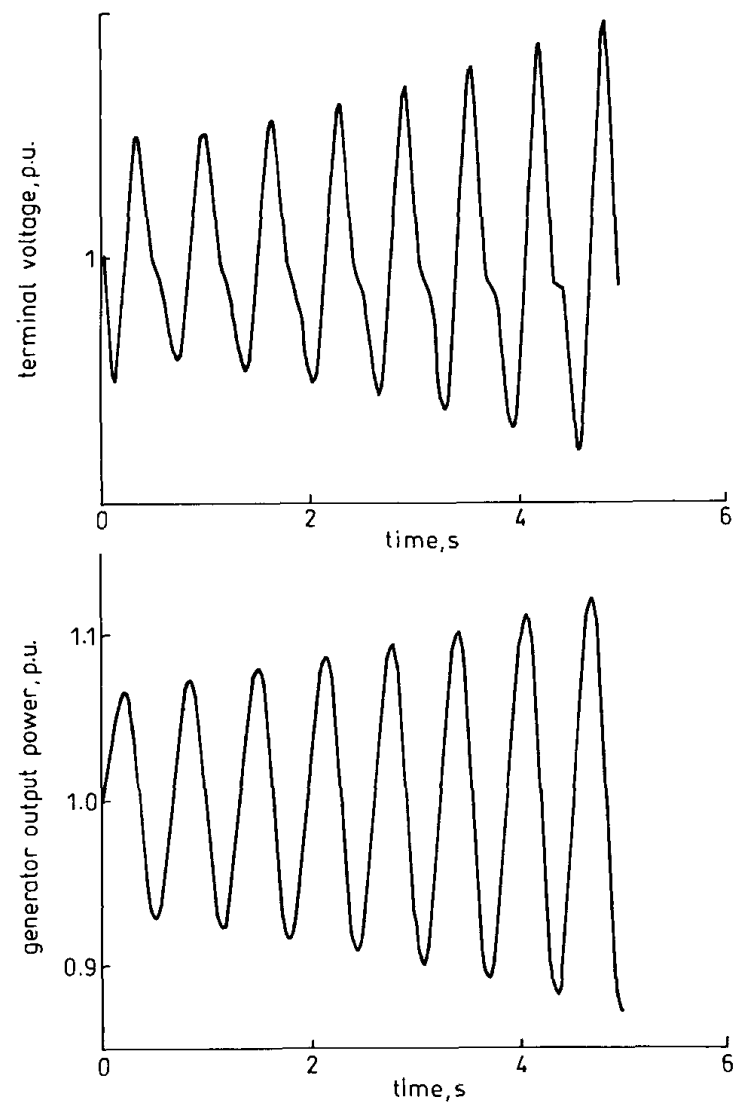

Fig. 4 Dynamic responses of generator without any controller $Q=0.6$

The two different design techniques explained previously are applied to design both the PSS and TCSC controllers. Time-domain simulations of the test system are performed under three different loading conditions, i.e. $P=1.0$ and $Q$ $=0.6, P=1.2$ and $Q=0.4$ and $P=0.85$ and $Q=0.4$. The response curves for these three cases are shown in Figs. 5 and 6,7 and 8,9 and 10 , respectively.

\section{Discussions}

From the results in Figs. 4-10, the following observations are made:

(a) As evidenced by the response curves in Fig. 4, the system without any controller is unstable. This is as expected since the electromechanical mode (characterised by the eigenvalues $0.135 \pm j 9.953$ in Table 1) has negative damping. 

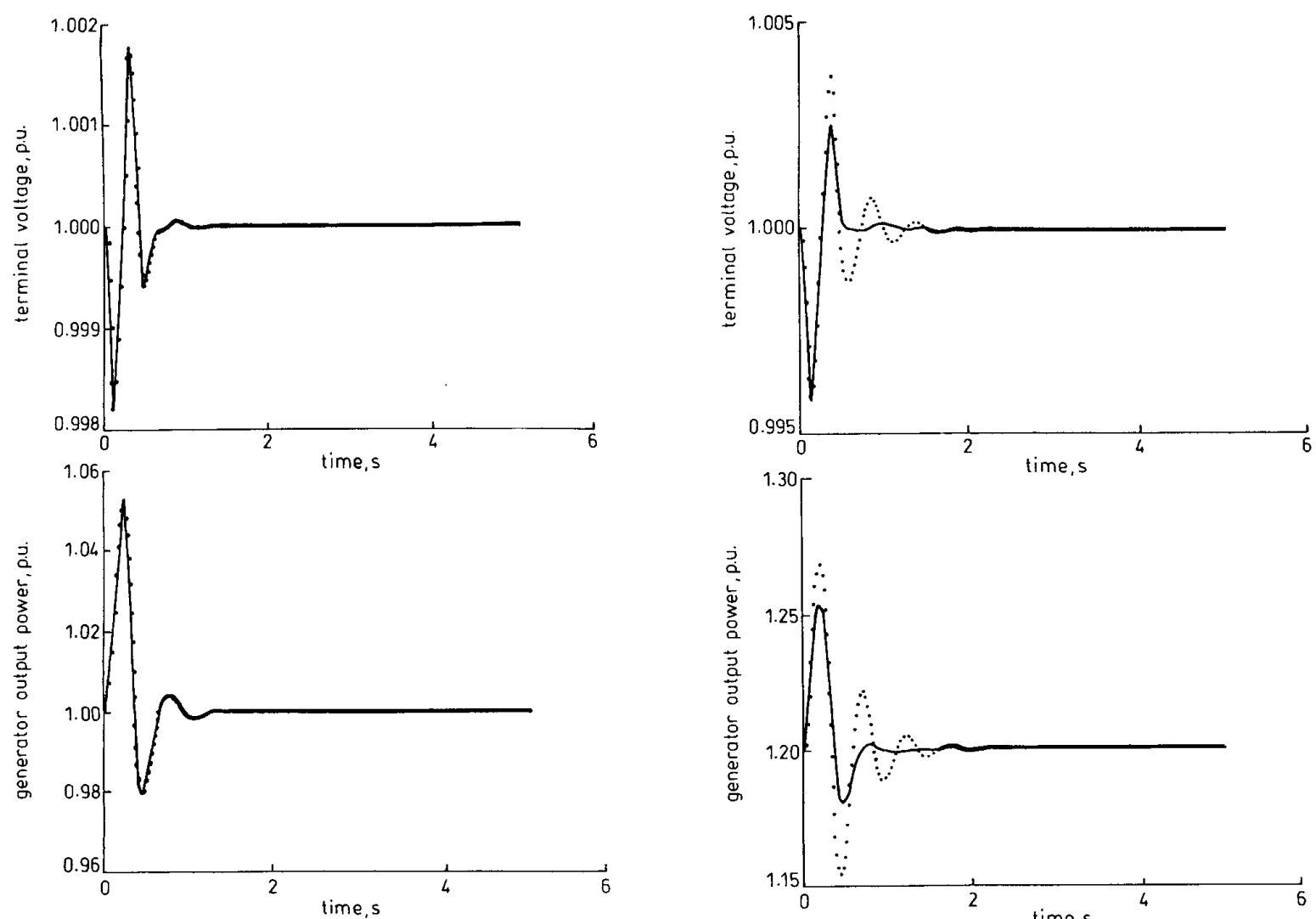

Fig.5 Dynamic responses of generator with controller

$P=1.0, Q=0.6$
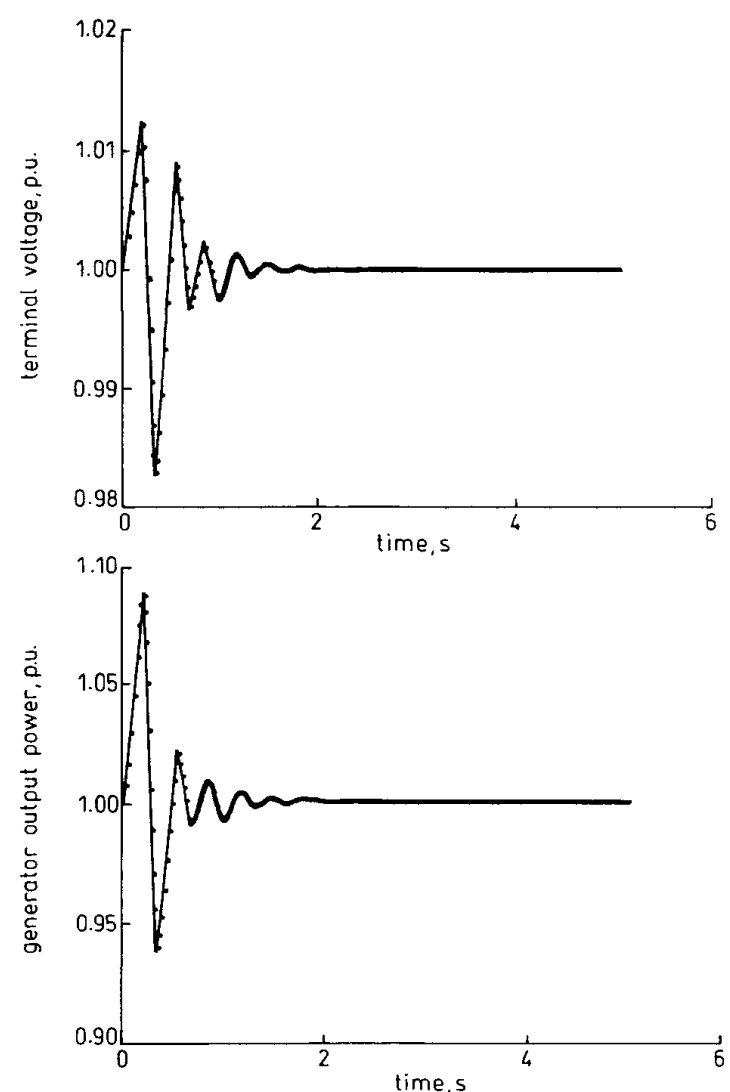

Fig. 6 Dynamic responses of generator with controller

$P=1.0, Q=0.6$ fixed-gain PSS _ adaptive PSS

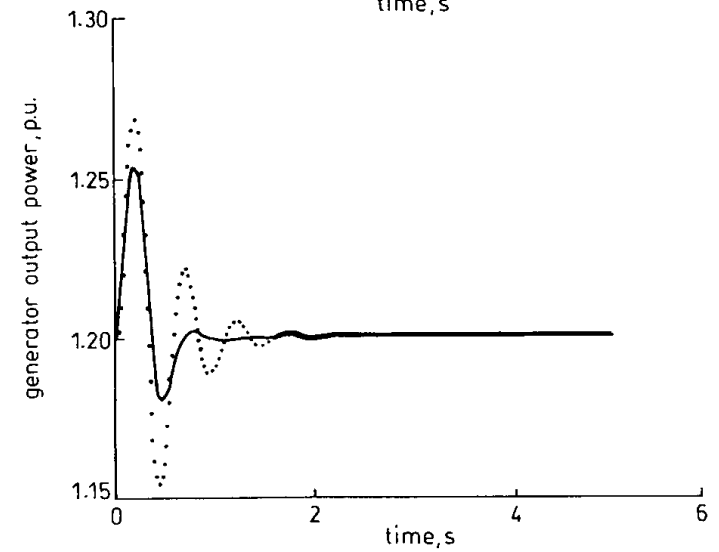

Fig. 7 Dynamic responses of generator with controller

$P=1.2, Q=0.4$, fixed-gain TCSC controller _ adaptive TCSC controller
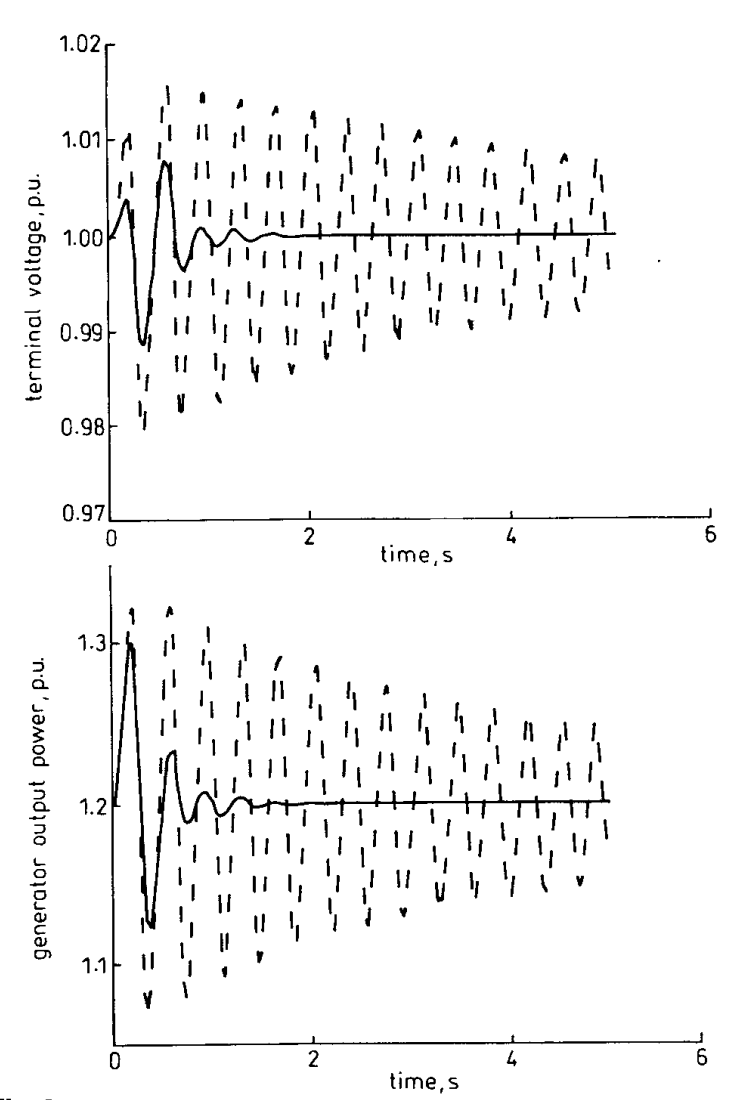

Fig. 8 Dynamic responses of generator with controller $P=1.2, Q=0.4$
--- fixed-gain PSS _— adaptive PSS

IEE Proc.-Gener. Transm. Distrib., Vol. 146, No. 2, March 1999 


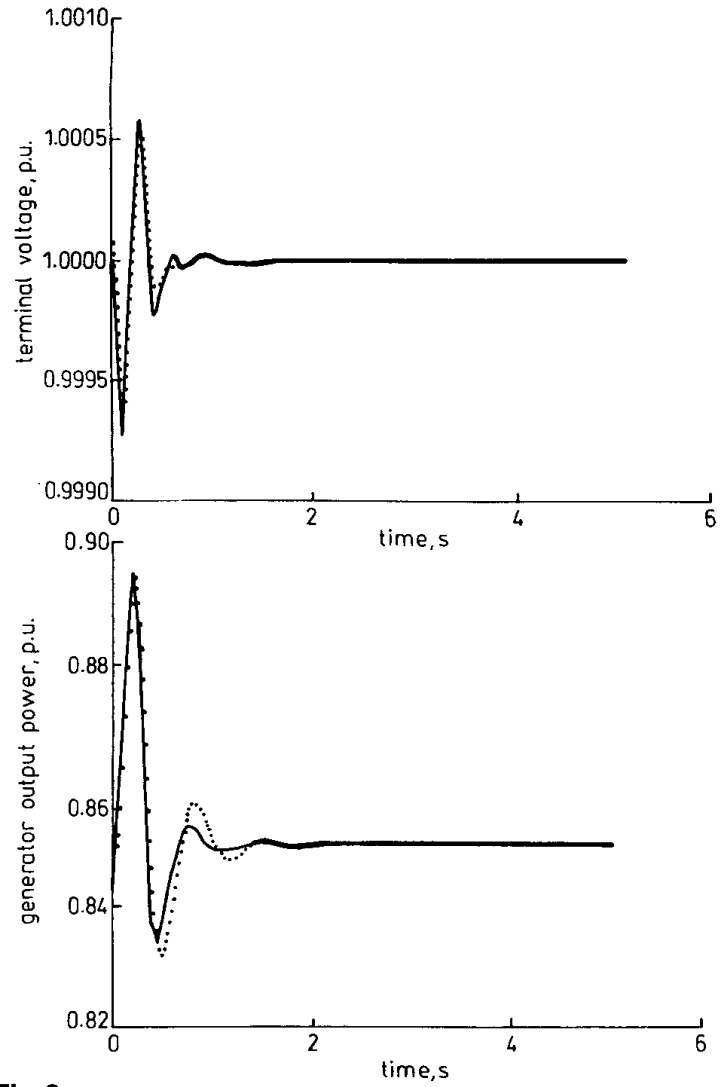

Fig.9 Dynamic responses of generator with controller $P=0.85, Q=0.4$
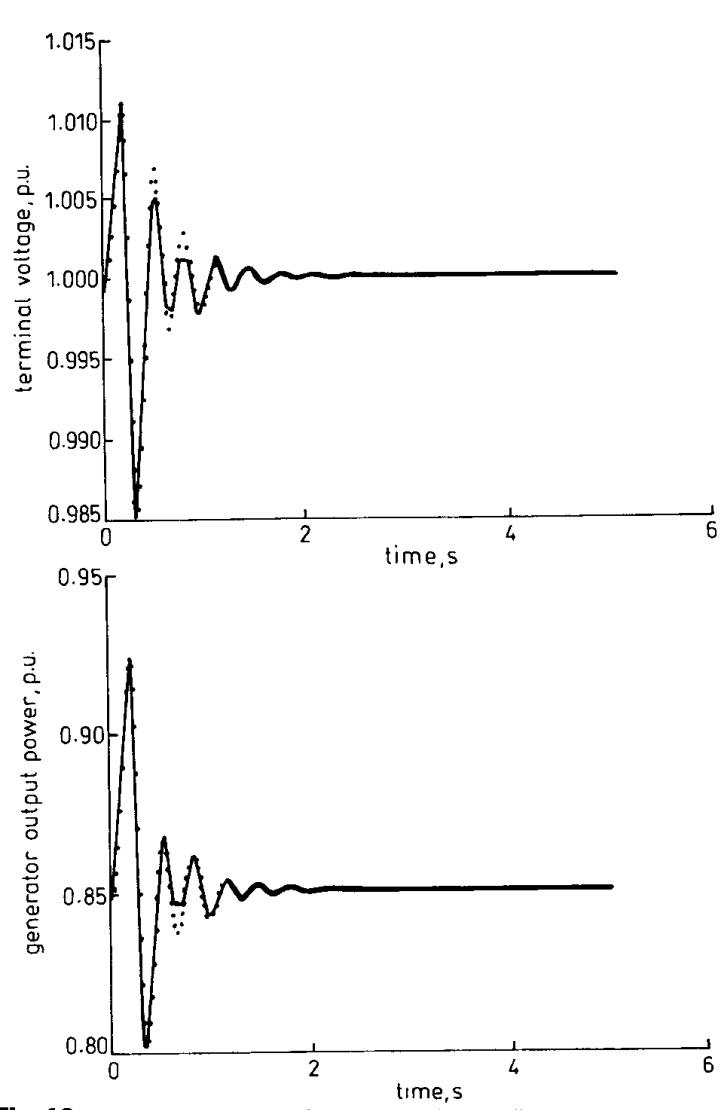

Fig. 10 Dynamic responses of generator with controller $P=0.85, Q=0.4 \quad$......... ${ }_{\text {fixed-gain PSS }}$

IEE Proc-Gener. Transm. Distrib., Vol. 146, No. 2, March 1999 (b) As shown in Fig. 5, the unstable electromechanical mode is damped as the fixed-gain proportional-integral TCSC controller is added. This is as expected since the PI TCSC controller will shift the eigenvalues for the mode from $0.135 \pm j 9.953$ to $-4.5 \pm j 10.681$ as shown in Table 1 . The installation of a PI PSS on the generator has a similar effect on the system, as shown by the response curves in Fig. 6.

(c) No significant difference is observed between the response curves from the fixed-gain TCSC controller and those from the adaptive controller in Fig. 5. This is as expected since the gains of the fixed-gain PI TCSC controller have been determined under the loading of $P=1.0$ and $Q=0.6$, which is exactly the operating point at which we are testing controller performance. In other words, the adaptive controller and the fixed-gain controller give similar response curves under the operating point at which the fixed-gain controller gains are determined. However, as the operating point changes, the damping for the system with a fixed-gain TCSC controller may deteriorate while a good damping effect can still be maintained in the system with an adaptive TCSC controller. This is evidenced by the response curves in Figs. 7 and 9, where better dynamic responses are observed for the system with the adaptive controller than for that with the fixed-gain controller.

(d) It is observed from Figs. 5-10 that the proposed ANN based adaptive TCSC controller not only improves the damping of system oscillations, which can be predicted by eigenvalues, but also improves the transient responses during the first swing period when the power system experiences highly nonlinear characteristics. However, since the capacity of TCSC is usually limited by economic considerations, the damping effect may be limited when the system is subject to a major disturbance such that the TCSC limit is reached.

(e) In the adaptive controller without ANN [17], system parameters must first be estimated by a recursive-leastsquares (RLS) method, which includes a rather time-consuming recursive process. Then the controller gains are computed using these parameters. Since these parameters change to a great extent during disturbance conditions, it is necessary to estimate these parameters online to have good damping effects. However, the ANN is trained offline and the adaptive controller with ANN does not require online estimation and training. Thus, the ANN based adaptive controller is more efficient in online applications than the adaptive controller without ANN.

(f) By comparing the response curves in Figs. 6, 8 and 10 for the system with PSS, it is also observed that the adaptive PSS can offer better dynamic responses than the fixedgain PSS, as the operating point changes from the nominal point at which we design the fixed-gain PI PSS.

(g) It is interesting to compare the responses from the TCSC and PSS in Figs. 5-10. The TCSC yields better dynamic responses than the PSS. This observation holds in either the fixed-gain controller case or the adaptive controller case. The difference is even more significant when we examine the terminal voltage response. In fact, relatively great voltage deviation is a major disadvantage of the PSS when it is used to stabilise low-frequency oscillations [20].

(h) The PSS is relatively cheaper than the TCSC. However, the TCSC is installed mainly to increase the power transfer capability of a transmission line. Once the TCSC is commissioned, stability enhancement can be achieved at little additional cost of the PI or adaptive damping controller. In systems which do not require transfer capability enhance- 
ment, it is sufficient to employ a PSS to improve system damping.

\section{Conclusions}

A novel tuning technique based on ANN is proposed to adapt the P1 controller gains of both TCSC and PSS to improve the dynamic performance of a power system. A major feature of the proposed adaptive TCSC controller is that only physically measurable real power and reactive power signals near the location of the TCSC are needed. The controller gains can be determined by the ANN in real time over a wide range of operating conditions. This makes the proposed TCSC controller relatively easy for practical implementation. Simulation results indicated that the proposed adaptive control scheme can provide an effective means for improving the damping characteristic of the power system.

\section{Acknowledgments}

Financial support given to this work by the National Science Council of R.O.C. under contract number NSC852213-E002-069 is appreciated.

\section{References}

1 EPRI EL-6943: 'Flexible AC transmission systems, FACTS, Scoping study', 1991

2 GYUGYI, L.: 'Unified power-flow control concept for flexible AC transmission system', IEE Proc., C, Gener. Transm. Distrib., 1992, 139, (4), pp. 323-331

3 PASERBA, J.J., MILLER, N.W., LARSEN, E.V., and PIWKO, R J.: "A thyristor controlled series compensation model for power system analysis' IEEE Trans, 1995, PD-10. (3) pp 1471-1478

4 ANGQUIST, L., LUNDIN, B.. and SAMUELSSON, J.: 'Power oscillation damping using controlled reactive power compensation - a comparison between series and shunt approaches', IEEE Trans., 1993, comparison between series

5 NOROOZIAN, M., and ANDERSSON, O.: 'Damping of power system oscillations by use of controllable components', IEEE Trans. 1994, PD-9, (4), pp. 2046-2053

6 NOROOZIAN, M., and ANDERSSON, G.: 'Damping of inter-area and local modes by use of controllable components', IEEE Trans., 1995, PD-10, (4), pp. 2007-2012
7 CHEN, X.R., PAHALAWATHTHA, N.C., ANNAKKAGE, U.D., and KUMBLE, C.S.: 'Controlled series compensation for improving the stability of multi-machine power systems', IEE Proc., Gener. Transm. Distrib., 1995, 142, (4), pp. 361-366

8 WANG, Y., MOHHER, R.R., SPEE, R., and MITTELSTADT, W. 'Variable-structure FACTS controllers for power system transient stability', IEEE Trans., 1992, PWRS-7, (1), pp. 307-313

9 LIE, T.T., SHRESTHA, G.B., and GHOSH, A.: 'Design and application of a fuzzy logic control scheme for transient stability enhancement in power systems', Electr. Power Syst. Res., 1995, 33, pp. 17-23

10 NOROOZIAN, M., ANDERSSON, G., and TOMSOVIC, K. 'Robust, near time-optimal control of power system oscillations with fuzzy logic', IEEE Trans., 1996, PD-11, (1), pp. 93-400

11 HIYAMA, T., MISHIRO, M., and KIHARA, H.: 'Coordinated fuzzy logic control for series capacitor modules and PSS to enhance stability of power system', IEEE Trans., 1995, PD-10, (2), pp. 10981104

12 LARSEN, E.V., SANCHEZ-GASCA, J.J., and CHOW, J.H.: 'Concepts for design of FACTS controller to damp power swings', IEEE Trans., 1995, PWRS-10, (2), pp. 948-955

13 DOLAN, P.S., SMITH, J.R. and MITTELSTADT, W.A. 'A study of TCSC optimal damping control parameters for different operating conditions', IEEE Trans., 1995, PWRS-10, (4), pp. 1972-1977

14 CHOI, S.S., JIANG, F., and SHRESTHA, G.: 'Suppression of transmission system oscillations by thyristor-controlled series compensations', IEE Proc. Gener. Transm. Distrib., 1996, 143, (1), pp. 7-12

15 DE MELLO, F.P.: 'Exploratory concepts on control of variable series compensation in transmission system to improve damping of intermachine/system oscillations', IEEE Trans., 1994, PWRS-9, (1), pp. 102 108

16 CHENG, C.H., and HSU, Y.Y.: 'Damping of generator oscillations using an adaptive static VAR compensator', IEEE Trans., 1992, PWRS-7, (2), pp. 718-725

17 HSU, Y.Y., and LIOU, K.L.: 'Design of self-tuning PID power system stabilizers for synchronous generators', IEEE Trans., 1987, EC-2. (3), pp. 343-348

18 HSU, Y.Y., and CHEN, C.R.: 'Tuning of power system stabilizers using an artificial neural network', IEEE Trans., 1991, EC-6, (4), pp. $612-619$

19 HSU, Y.Y., and JENG, L.H.: 'Damping of subsynchronous oscillations using adaptive controllers tuned by artificial neural networks', IEE Proc. Gener. Transm. Distrib., 1995, 142, (4), pp. 415-422

20 ANDERSON, P.M., and FOUAD, A.A.: 'Power system control and stability' (Iowa State University Press, 1977)

21 PORTER, B., and CROSSLEY, R.: 'Modal control theory and applications' (Taylor and Francis, 1972)

22 EURADA, J.M.: 'Introduction to artificial neural systems' (West Publishing Company, 1992)

23 HSU, Y.Y., and JENG, L.H.: 'Analysis of torsional oscillations using an artificial neural networks', IEEE Trans., 1992, EC-7, (4), pp. 684 690 